\title{
-1 V Bias 56 Gbps Germanium Waveguide p-i-n Photodetector with Silicon Contacts
}

\author{
H. Chen ${ }^{1,2}$, P. Verheyen ${ }^{1}$, P. De Heyn', G. Lepage ${ }^{1}$, J. De Coster ${ }^{1}$, P. Absil ${ }^{1}$, \\ G. Roelkens ${ }^{2}$, and J. Van Campenhout ${ }^{1}$ \\ ${ }^{1}$ Imec, Kapeldreef 75, Leuven B-3001, Belgium \\ ${ }^{2}$ Photonics Research Group, Department of Information Technology, Ghent University-IMEC, B-9000 Ghent, Belgium \\ hongtao.chen@imec.be
}

\begin{abstract}
We demonstrate a silicon-contact-only 56 Gbps germanium waveguide photodetector operating at $-1 \mathrm{~V}$. The dark current is below $4 \mathrm{nA}$ and the responsivity is $0.74 \mathrm{~A} / \mathrm{W}$ at $1550 \mathrm{~nm}$ and $0.93 \mathrm{~A} / \mathrm{W}$ at $1310 \mathrm{~nm}$.

OCIS codes: (230.5160) Photodetectors; (130.3120) Integrated optics devices; (200.4650) Optical interconnects.
\end{abstract}

\section{Introduction}

A germanium waveguide $\mathrm{p}-\mathrm{i}-\mathrm{n}$ photodetectors (WPD) is a critical building block in silicon photonics optical interconnects and has been researched extensively [1-9]. Conventional germanium WPDs require doping in germanium as well as a metal contact on germanium to form the p-i-n junction. This is sub-optimal as germanium processing is less well understood and characterized. Besides, light absorption from metal contacts on germanium is responsible for a substantial responsivity loss in these devices. We have demonstrated a germanium WPD that does not require doping or contacting of germanium (named Si-LPIN GePD hereafter) showing a very high responsivity over $1 \mathrm{~A} / \mathrm{W}$ across the whole C-band and a very low dark current of $3 \mathrm{nA}$ at $-1 \mathrm{~V}$ [7]. However, the opto-electrical 3-dB bandwidth of the device was transit-time limited to $20 \mathrm{GHz}$ at $-1 \mathrm{~V}$.

In this paper, by optimizing the device design and adopting a $160 \mathrm{~nm}$ thin germanium layer to reduce the transit time, the opto-electrical $3-\mathrm{dB}$ bandwidth at $-1 \mathrm{~V}$ is enhanced to over $50 \mathrm{GHz}$ and $44 \mathrm{GHz}$ at $1550 \mathrm{~nm}$ and $1310 \mathrm{~nm}$, respectively. The measured responsivity at $-1 \mathrm{~V}$ is $0.74 \mathrm{~A} / \mathrm{W}$ and $0.93 \mathrm{~A} / \mathrm{W}$ at $1550 \mathrm{~nm}$ and $1310 \mathrm{~nm}$. The dark current is below $4 \mathrm{nA}$ at $-1 \mathrm{~V}$. 56 Gbps on-off key data reception is demonstrated with clear open eye diagrams.

\section{Device Design and Fabrication}

The Si-LPIN GePDs were fabricated in imec's fully integrated Si photonics platform along with Si modulators and various passive devices, going through a process flow described in [10]. Light is coupled from a $220 \mathrm{~nm}$ thick single-mode Si waveguide to the Ge layer using a fully etched waveguide taper together with a $120 \mathrm{~nm}$ thick poly-Si taper above, as shown in Fig. 1(a). The Ge layer dimensions and doping configuration are shown in Fig. 1(b). The electric field distribution in the Si-LPIN GePD at $-1 \mathrm{~V}$ obtained by numerically solving Poisson's equation is shown in Fig. 1(c). In the germanium region, the electric field is stronger than $10^{4} \mathrm{~V} / \mathrm{cm}$ at $-1 \mathrm{~V}$, strong enough for photogenerated carriers to drift at the saturation velocity. In [7], where a $400 \mathrm{~nm}$ thick germanium layer was used, the $\mathrm{O} / \mathrm{E}$ bandwidth was limited by the long transit time of the photo-carriers generated in the top part of the germanium, suffering from low drift velocity as well as long drift distance. In the current device, this bandwidth limitation is eliminated by using a $160 \mathrm{~nm}$ thick germanium layer. Therefore, a much higher O/E bandwidth can be expected.
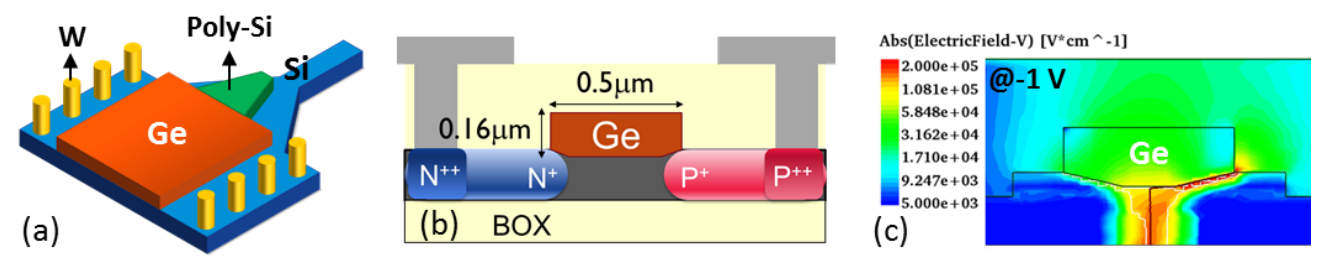

Fig. 1. (a) 3-D schematic of the Si-LPIN GePD. (b) Cross-section schematic of the Si-LPIN GePD. (c) Simulated electric field distribution in the Si-LPIN GePD at $-1 \mathrm{~V}$.

\section{Device Characteristics}

A typical static current-voltage characteristic of a $14 \mu \mathrm{m}$ long and $0.5 \mu \mathrm{m}$-wide Si-LPIN GePD is shown in Fig. 2(a). The device has a low dark current of $2.5 \mathrm{nA}$ at $-1 \mathrm{~V}$. The photocurrent was measured in the C-band at $1550 \mathrm{~nm}$ and in the O-band at $1310 \mathrm{~nm}$ with a received optical power of $-5.8 \mathrm{dBm}$ and $-2.5 \mathrm{dBm}$, respectively. The photocurrent is almost constant from $0 \mathrm{~V}$ to $-2 \mathrm{~V}$ owing to the strong built-in electric field that is capable of sweeping out the majority of photo-generated carriers within their lifetime. The measured responsivity at $-1 \mathrm{~V}$ is $0.72 \mathrm{~A} / \mathrm{W}$ and 0.98 $\mathrm{A} / \mathrm{W}$ at $1550 \mathrm{~nm}$ and $1310 \mathrm{~nm}$, respectively. The wavelength dependence of the responsivity in the C-band and O- 
band at $-1.2 \mathrm{~V}$ are shown in Fig. 2(b) and Fig. 2(c), respectively. Only responsivity data in $40 \mathrm{~nm}$ optical bandwidth are shown limited by the bandwidth of the fiber grating coupler. The device has a higher responsivity in the O-band than that in the C-band due to the higher mode confinement factor in the Ge layer in the O-band. The drop in responsivity in the $\mathrm{C}$-band is due to the decrease of the Ge absorption coefficient at longer wavelength, which can be improved by increasing the length of the photodetector.
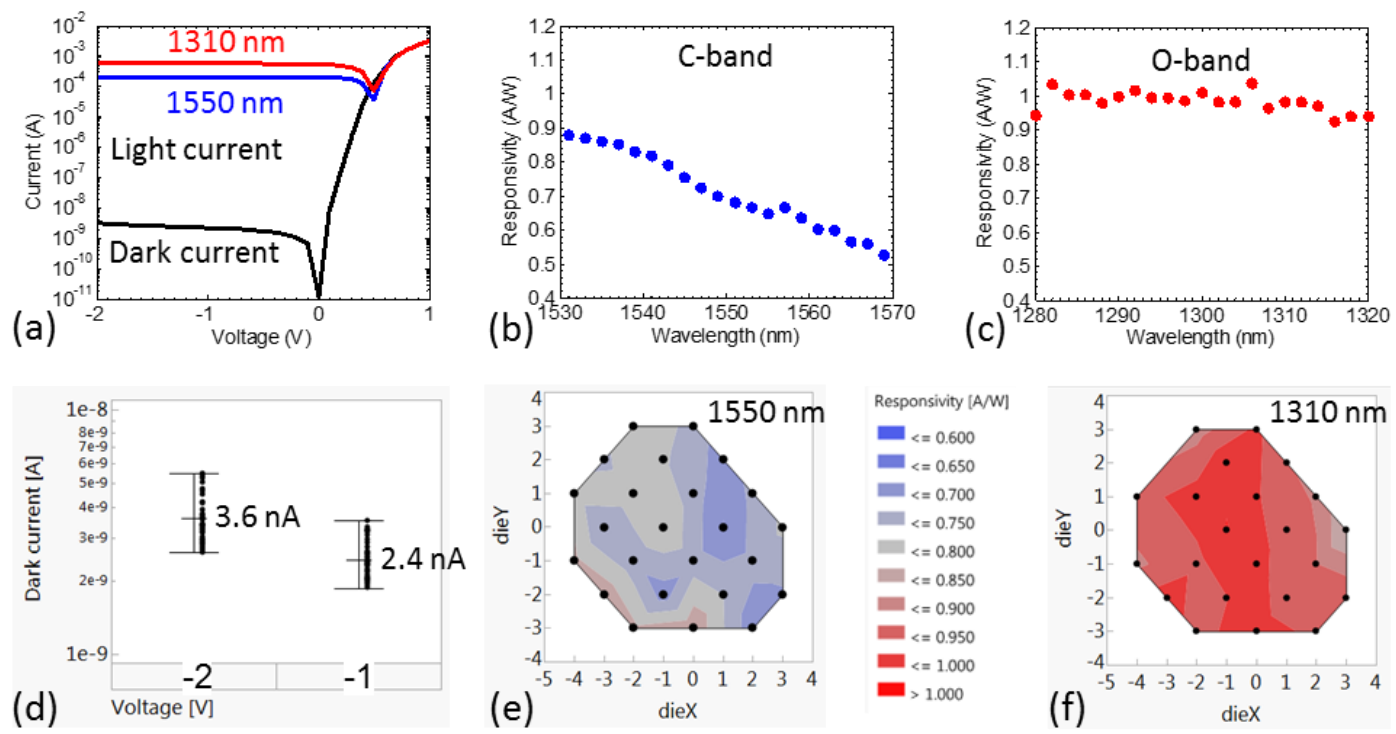

Fig. 2. (a) I-V characteristics of a Si-LPIN GePD. Responsivity of the Si-LPIN GePD in (b) the C-band and (c) the O-band at -1.2 V. (d) Waferscale dark current data of the Si-LPIN GePD. Wafer-scale responsivity data of the Si-LPIN GePD at (e) $1550 \mathrm{~nm}$ and (f) $1310 \mathrm{~nm}$ at $-1 \mathrm{~V}$.
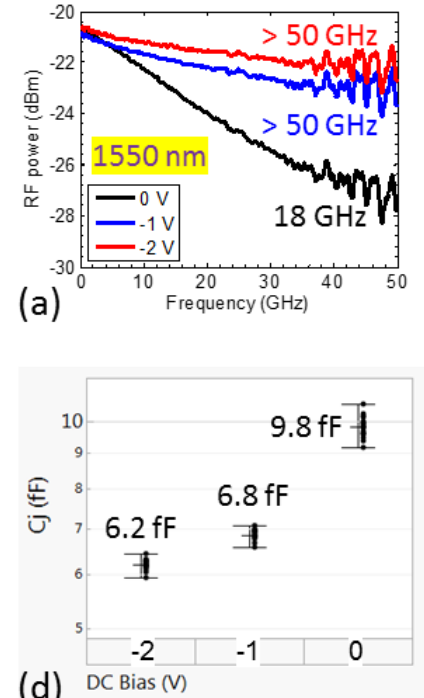

(d)
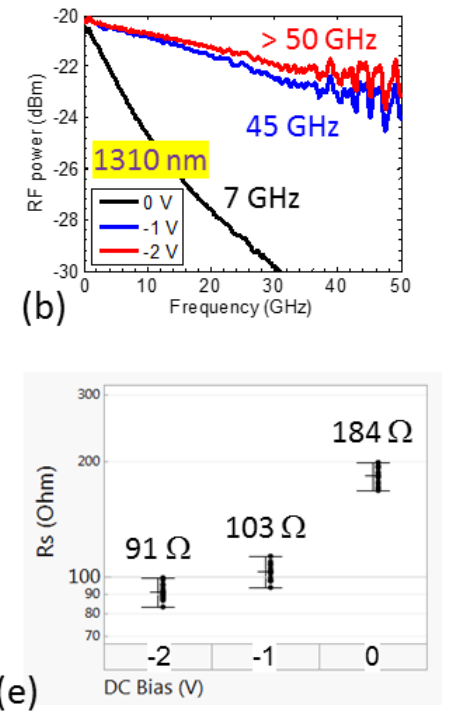

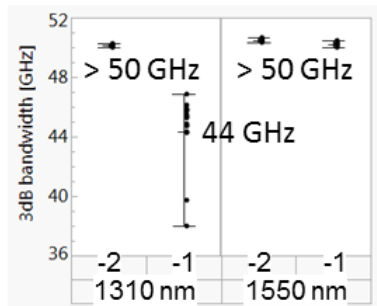

(C) DC Bias [V] within Wavelength [nm]

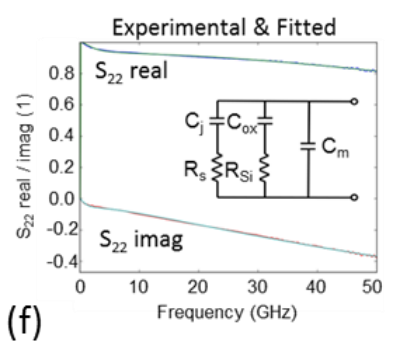

Fig. 3. Small-signal $\mathrm{S}_{21}$ traces of the Si-LPIN GePD at (a) $1550 \mathrm{~nm}$ and (b) $1310 \mathrm{~nm}$. (c) Wafer-scale O/E 3-dB bandwidth data. (d) Fitted junction capacitance data. (e) Fitted series resistance data. (f) Experimental and fitted real/imaginary part of the $S_{22}$ parameter. The inset is the equivalent circuit model used for the fitting.

Wafer-scale dark current data of the Si-LPIN GePD are shown in Fig. 2(d). The mean dark current value is 2.4 $\mathrm{nA}$ and $3.6 \mathrm{nA}$, with a standard deviation of $0.4 \mathrm{nA}$ and $0.8 \mathrm{nA}$, at $-1 \mathrm{~V}$ and $-2 \mathrm{~V}$, respectively. In Fig. 2(e) and (f), contour plots of the wafer-scale responsivity data of the Si-LPIN GePD at $-1 \mathrm{~V}$ at $1550 \mathrm{~nm}$ and $1310 \mathrm{~nm}$ are shown. The mean responsivity value is $0.74 \mathrm{~A} / \mathrm{W}$ and $0.93 \mathrm{~A} / \mathrm{W}$ respectively, with a standard deviation of $0.05 \mathrm{~A} / \mathrm{W}$.

Small-signal radio-frequency (RF) measurements were further carried out to characterize the high-speed performance of the Si-LPIN GePD. $S_{21}$ traces measured at $1550 \mathrm{~nm}$ and $1310 \mathrm{~nm}$ using an average optical power of $-6 \mathrm{dBm}$ and $-5 \mathrm{dBm}$ are shown in Fig. 3(a) and Fig. 3(b), respectively. At $-1 \mathrm{~V}$, the device has a 3-dB bandwidth of 
over $50 \mathrm{GHz}$ and $45 \mathrm{GHz}$ at $1550 \mathrm{~nm}$ and $1310 \mathrm{~nm}$, sufficient for receiving $56 \mathrm{~Gb} / \mathrm{s} \mathrm{NRZ-OOK}$ signals. The bandwidth is further enhanced to over $50 \mathrm{GHz}$ for both wavelengths as the bias voltage increases to $-2 \mathrm{~V}(50 \mathrm{GHz}$ is the upper limit of the vector network analyzer measurement range). Wafer-scale O/E 3-dB bandwidth data for both wavelengths are shown in Fig. 3(c). At $1550 \mathrm{~nm}$, the wafer-scale 3-dB bandwidth is over $50 \mathrm{GHz}$ at both $-1 \mathrm{~V}$ and $2 \mathrm{~V}$. At $1310 \mathrm{~nm}$, the wafer-scale $3-\mathrm{dB}$ bandwidth is over $50 \mathrm{GHz}$ at $-2 \mathrm{~V}$. At $-1 \mathrm{~V}$, the mean $3-\mathrm{dB}$ bandwidth value is $44 \mathrm{GHz}$ with a standard deviation of $2.2 \mathrm{GHz}$.

The capacitance of the Si-LPIN GePD was extracted by fitting the real/imaginary part of the wafer-scale smallsignal reflection $\left(\mathrm{S}_{22}\right)$ parameter based on the equivalent circuit model shown in the inset of Fig. 3(f). In the circuit model, $C_{j}$ and $R_{s}$ are related to the reverse biased p-i-n junction, while $C_{O X}$ and $R_{S i}$ are related to the current path through the silicon substrate and the buried oxide (BOX). $\mathrm{C}_{\mathrm{m}}$ represents the metal pad capacitance. The extracted junction capacitance $\left(\mathrm{C}_{\mathrm{j}}\right)$ data and series resistance $\left(\mathrm{R}_{\mathrm{s}}\right)$ data of the Si-LPIN GePD are shown in Fig. 3(d) and Fig. 3(e). The mean junction capacitance value is $6.8 \mathrm{fF}$ and $6.2 \mathrm{fF}$ at $-1 \mathrm{~V}$ and $-2 \mathrm{~V}$. The mean series resistance value is $103 \Omega$ and $91 \Omega$ at $-1 \mathrm{~V}$ and $-2 \mathrm{~V}$. One example of the experimental and fitted real/imaginary part of the $\mathrm{S}_{22}$ parameter are shown in Fig. 3 (f).

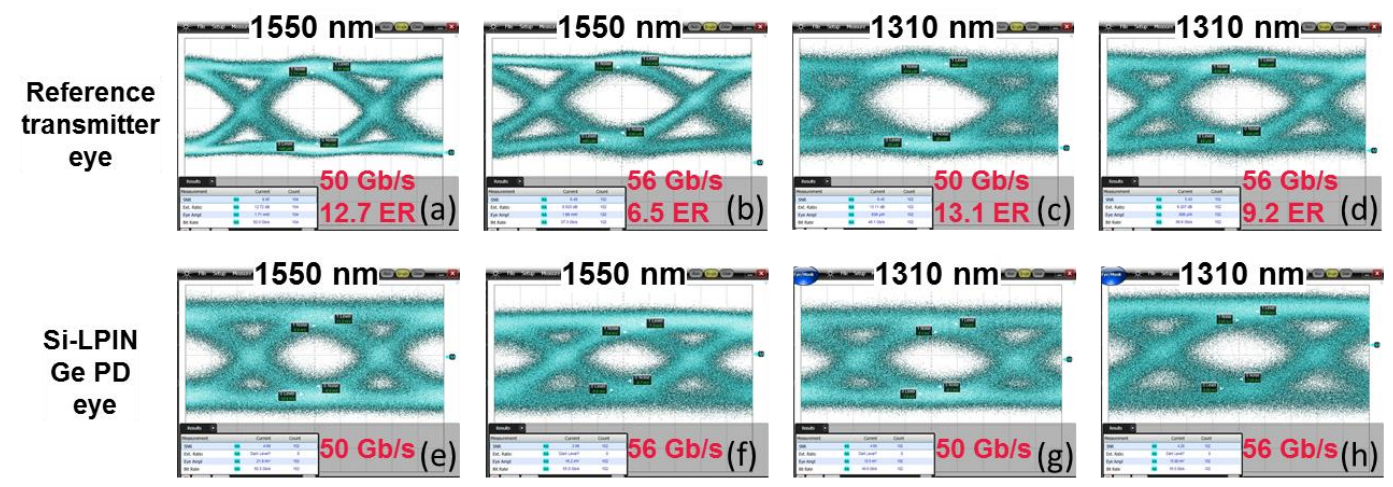

Fig. 4. Optical eye generated (a) at $1550 \mathrm{~nm}, 50 \mathrm{Gbps}$, (b) at $1550 \mathrm{~nm}, 56 \mathrm{Gbps}$, (c) at $1310 \mathrm{~nm}, 50 \mathrm{Gbps}$ and (d) at $1310 \mathrm{~nm}, 56 \mathrm{Gbps}$. The corresponding electrical eye from the Si-LPIN GePD at $-1 \mathrm{~V}$ bias voltage is shown in (e-h).

On-off key data transmission experiments were carried out. A $\left(2^{31}-1\right)$ long optical non-return to zero (NRZ) pseudo random bit sequence (PRBS) data pattern at $50 \mathrm{Gbps}$ and $56 \mathrm{Gbps}$, generated by a $\mathrm{LiNbO}_{3}$ modulator at $1550 \mathrm{~nm}$ and $1310 \mathrm{~nm}$ was launched into the Si-LPIN GePD. A -1 V bias voltage was applied to the photodetector using a $50 \mathrm{GHz}$ RF probe connected to a $40 \mathrm{GHz}$ bias-tee. The photocurrent output was measured with an Agilent oscilloscope with a $60 \mathrm{GHz}$ remote sampling head plug-in. The reference transmitter eye at $50 \mathrm{Gbps}$ and $56 \mathrm{Gbps}$ at $1550 \mathrm{~nm}$ and $1310 \mathrm{~nm}$ wavelength is shown in Fig. 4(a-d). The Si-LPIN GePD eye at $-1 \mathrm{~V}$ is shown in Fig. 4(e-h). The clear open eye diagrams from the Si-LPIN GePD at $-1 \mathrm{~V}$ indicate high quality data reception of the Si-LPIN GePD at $56 \mathrm{Gbps}$, both in the C-band and O-band.

\section{Conclusion}

A 56 Gbps germanium waveguide p-i-n photodetector has neither doping in nor metal contacts on germanium operating at $-1 \mathrm{~V}$ is reported. The device was characterized in both the C-band and O-band with high responsivity and low dark current.

This work was carried out as part of imec's industry affiliation program on Optical I/O. The device simulations were performed in Sentaurus TCAD, provided by Synopsys. The device layout was performed in IPKISS provided by Luceda Photonics. We acknowledge imec's mask preparation team and process line for their contributions.

[1] T. Yin, et al. "31 GHz Ge n-i-p waveguide photodetectors on Silicon on-Insulator substrate," Opt. Exp., 15(21), 13965 (2007).

[2] L. Vivien, et al. "42 GHz p.i.n Germanium photodetector integrated in a silicon-on-insulator waveguide," Opt. Exp., 17(8), 6252 (2009).

[3] D. Feng, et al. "High-speed Ge photodetector monolithically integrated with large cross-section silicon-on-insulator waveguide," Appl. Phys. Lett., 95(26), 261105 (2009).

[4] C. T. DeRose, et al. "Ultra-compact $45 \mathrm{GHz}$ CMOS compatible Germanium waveguide photodiode with low dark current," Opt. Exp., 19 (25), 24897 (2011).

[5] A. Novack, et al., "Germanium photodetector with $60 \mathrm{GHz}$ bandwidth using inductive gain peaking," Opt. Exp., 21 (23), 28387 (2013).

[6] Y. Zhang, et al. "A high responsivity photodetector absent metal-germanium direct contact," Opt. Exp., 22 (9), 11367 (2014).

[7] H. Chen, et al. "High responsivity low-voltage 28Gb/s Ge p-i-n photodetector with silicon contacts," J. Lightwave Technol., 2367134 (2014).

[8] R. Going, et al. "Germanium wrap-around photodetectors on Silicon photonics," Opt. Exp., 23 (9), 11975 (2015).

[9] S. Lischke, et al. "High bandwidth, high responsivity waveguide-coupled germanium p-i-n photodiode," Opt. Exp., 23 (21), 27213 (2015).

[10] P. Verheyen, et al. "Highly uniform $25 \mathrm{~Gb} / \mathrm{s}$ Si photonics platform for high-density, low-power WDM optical interconnects," presented at the Integr. Photon. Res., Silicon Nanophoton. Conf., 2014, Paper IW3A.4. 